Research Article

\title{
Successive Linearization Analysis of the Effects of Partial Slip, Thermal Diffusion, and Diffusion-Thermo on Steady MHD Convective Flow due to a Rotating Disk
}

\author{
S. S. Motsa ${ }^{1}$ and S. Shateyi ${ }^{2}$ \\ ${ }^{1}$ School of Mathematical Sciences, University of KwaZulu-Natal, Private Bag X01, Pietermaritzburg, \\ Scottsville 3209, South Africa \\ ${ }^{2}$ Department of Mathematics, University of Venda, P Bag X5050, Thohoyandou 0950, South Africa
}

Correspondence should be addressed to S. Shateyi, stanford.shateyi@univen.ac.za

Received 21 February 2012; Revised 7 May 2012; Accepted 15 May 2012

Academic Editor: P. Liatsis

Copyright $@ 2012$ S. S. Motsa and S. Shateyi. This is an open access article distributed under the Creative Commons Attribution License, which permits unrestricted use, distribution, and reproduction in any medium, provided the original work is properly cited.

\begin{abstract}
We proposed a general formulation of the successive linearization method for solving highly nonlinear boundary value problem arising in rotating disk flow. The problem was studied under the effects of partial slip, thermal diffusion, and diffusion-thermo. The governing fundamental conservation equations of mass, momentum, angular momentum, energy, and concentration are transformed into a system of ordinary differential equations by means of similarity transformations. A parametric study illustrating the influence of the magnetic field, slip factor, Eckert number, Dufour and Soret numbers was carried out.
\end{abstract}

\section{Introduction}

The growing concern of global warming effects of convectional fossil fuels forces energy research into the direction of the renewable energy sources, like in the design of turbines and turbomachines. The energy, chemical, and automobile industries extensively use disk-shaped bodies which are often encountered in many engineering applications and heat transfer problem of natural convection boundary layer flow over a rotating disk, which occurs in rotating heat exchangers, rotating disks for biofuels production and turbines.

In his pioneering work, von Karman [1] considered the case of an infinite disk and gave a formulation of the hydrodynamic problem. Since then, a lot of work has been done in this field of study (see, e.g., Cochran [2]; Benton [3]; Attia [4]; Miklavčič and Wang [5]; Arikoglu and Ozkol [6], among others). 
The relations between the fluxes and driving potentials are more intricate in nature, when heat and mass transfer occurs simultaneously in a moving fluid. It is now a known fact that an energy flux can be generated not only by temperature gradients but also by composition gradients. The energy flux caused by composition gradient is called the diffusion thermal or Dufour effect. Temperature gradients can also create mass fluxes. This phenomenon is called the thermal diffusion or Soret effect.

Generally, Soret and Dufour effects are of smaller-order magnitude than the effects prescribed by Fourier's or Fick's laws and are often neglected in heat and mass transfer processes. However, there are exceptions. The Soret effect plays an important role in the operation of solar ponds, biological systems, and the microstructure of the world oceans. On the other hand Dufour effect was found to be of order of considerable magnitude in mixture between gasses with very low molecular weight $\left(\mathrm{He}, \mathrm{H}_{2}\right)$ and of medium molecular weight ( $\mathrm{N}_{2}$, air), (Kafoussias and Williams [7]).

Siginer [8] observed that the Soret effect plays an important role in the concentration distribution of different components in hydrocarbon mixtures as these are mainly driven by phase separation and diffusion.

Osalusi et al. [9] investigated Soret and Dufour effects on combined heat and mass transfer of steady hydromagnetic convective and slip flow due to a rotating disk in the presence of viscous dissipation and ohmic heating. Rashidi et al. [10] solved the same problem analytically using the Homotopy analysis method. Turkyilmazoglu [11] derived analytical expressions for the solution of steady, laminar, incompressible, viscous fluid of the boundary layer flow due to a rotating disk in the presence of a uniform suction/injection. Shateyi et al. [12] investigated the influence of a magnetic field on heat and mass transfer by mixed convection from vertical surfaces in the presence of Hall, radiation, Soret and Dufour effects. Alam and Ahammad [13] investigated the effects of variable chemical reaction and variable electric conductivity on free convection flow with heat and mass transfer over an inclined permeable stretching sheet under the influence of Dufour and Soret effects with variable heat and mass fluxes.

Srinivasacharya and Kaladhar [14] employed the Homotopy analysis method to investigate the Hall and ion-slip effects on steady free convective heat transfer flow between two cylinders in couple stress fluid flow. Pal and Mondal [15] carried out an analysis of the combined effects of Soret and Dufour on unsteady MHD non-Darcy mixed convection over a stretching sheet embedded in a saturated porous medium in the presence of thermal radiation, viscous dissipation, and first-order chemical reaction.

More improved models which more accurately predict the concentrations of the different components of the fluid physics in crude oil reservoirs are necessary. Through the advancement of the symbolic computation software such as MATHEMATICA, MAPLE, and MATLAB, approximate analytic methods for nonlinear problems have been adopted by many researchers. Among these are homotopy perturbation method, Dehghan et al. [16], homotopy analysis method, Liao [17], Dehghan and Salehi [18], spectral homotopy analysis method, Motsa et al. [19], improved spectral homotopy analysis method, Motsa et al. [20], and successive linearization method (SLM), Motsa and Shateyi [21].

In this work we present a general formulation of the successive linearization method that can be used to solve any two-point nonlinear boundary value problem. We test the validity of the method of solution on the model of steady MHD convective flow due to a rotating disk with partial slip in the presence of thermal diffusion and diffusion-thermo effects. The objective of this study is to demonstrate the ease of use of the SLM approach and its accuracy when solving nonlinear BVPs arising in rotating disk flow. 


\section{Problem Formulation}

The problem of steady hydromagnetic convective and slip flow due to a rotating disk in the presence of viscous dissipation and ohmic heating is considered in this study. The effects of thermal diffusion, diffusion-thermo, and heat and mass transfer are also considered in the present study. The disk rotates at $z=0$ with constant angular velocity $\Omega$. We have $z$ being the vertical axis in the cylindrical coordinates system with $r$ and $\phi$ as the radial and tangential axes, respectively. The surface of the rotating disk is maintained at a uniform temperature $T_{w}$ and uniform concentration $C_{w}$. Far away from the surface, the free stream is kept at a constant temperature $T_{\infty}$, concentration $C_{\infty}$, and pressure $P_{\infty}$. The viscous fluid is an electrically conducting one. A uniform magnetic field is applied normally to the surface of the disk and has a constant magnetic flux density $B_{0}$ which is assumed unchanged by taking small magnetic Reynolds number. In this study we also assume that there is no applied electric field (Rashidi et al. [10]) and that the Hall effects are negligible. The equations governing the motion of MHD laminar flow take the following form:

$$
\begin{gathered}
\frac{\partial u}{\partial r}+\frac{u}{r}+\frac{\partial w}{\partial z}=0, \\
u \frac{\partial u}{\partial r}-\frac{v^{2}}{r}+w \frac{\partial u}{\partial z}+\frac{1}{\rho} \frac{\partial P}{\partial r}=v\left(\frac{\partial^{2} u}{\partial r^{2}}+\frac{1}{r} \frac{\partial u}{\partial r}-\frac{u}{r^{2}}+\frac{\partial^{2} u}{\partial z^{2}}\right)-\frac{\sigma B_{0}^{2} u}{\rho}, \\
u \frac{\partial v}{\partial r}+\frac{u v}{r}+w \frac{\partial v}{\partial z}=v\left(\frac{\partial^{2} v}{\partial r^{2}}+\frac{1}{r} \frac{\partial v}{\partial r}-\frac{v}{r^{2}}+\frac{\partial^{2} v}{\partial z^{2}}\right)-\frac{\sigma B_{0}^{2} v}{\rho}, \\
u \frac{\partial w}{\partial r}+w \frac{\partial w}{\partial z}+\frac{1}{\rho} \frac{\partial P}{\partial z}=v\left(\frac{\partial^{2} w}{\partial r^{2}}+\frac{1}{r} \frac{\partial w}{\partial r}+\frac{\partial^{2} w}{\partial z^{2}}\right) \\
u \frac{\partial T}{\partial r}+w \frac{\partial T}{\partial z}=\frac{\kappa}{\rho c_{p}}\left(\frac{\partial^{2} T}{\partial r^{2}}+\frac{1}{r} \frac{\partial T}{\partial r}+\frac{\partial^{2} T}{\partial z^{2}}\right)+\frac{\sigma B_{0}^{2}}{\rho c_{p}}\left(u^{2}+v^{2}\right) \\
+\frac{\mu}{\rho c_{p}}\left[\left(\frac{\partial u}{\partial z}\right)^{2}+\left(\frac{\partial v}{\partial z}\right)^{2}\right]+\frac{D k_{T}}{c_{s} c_{p}}\left(\frac{\partial^{2} C}{\partial r^{2}}+\frac{1}{r} \frac{\partial C}{\partial r}+\frac{\partial^{2} C}{\partial z^{2}}\right), \\
u \frac{\partial C}{\partial r}+w \frac{\partial C}{\partial z}=D\left(\frac{\partial^{2} C}{\partial r^{2}}+\frac{1}{r} \frac{\partial C}{\partial r}+\frac{\partial^{2} C}{\partial z^{2}}\right)+\frac{D k_{T}}{T_{m}}\left(\frac{\partial^{2} T}{\partial r^{2}}+\frac{1}{r} \frac{\partial T}{\partial r}+\frac{\partial^{2} T}{\partial z^{2}}\right),
\end{gathered}
$$

where $u, v, w$ are the velocity components of the flow in the directions of increasing $(r, \phi, z)$, respectively, $P$ is the pressure, $\rho$ is the fluid density. $T$ and $C$ are the fluid temperature and concentration, respectively. $v=\mu / \rho$ is the kinematic viscosity of the ambient fluid, $\kappa$ is the thermal conductivity, $\sigma$ is the electrical conductivity, $c_{p}$ is the specific heat at constant pressure, $D$ is the molecular diffusion coefficient, $k_{T}$ is the thermal-diffusion ratio, $c_{s}$ is the concentration susceptibility, and $T_{m}$ is the mean fluid temperature. 
The boundary conditions for the model can be expressed as

$$
\begin{gathered}
u=\frac{2-\xi}{\xi} \lambda \frac{\partial u}{\partial z}, \quad v=r \Omega+\frac{2-\xi}{\xi} \lambda \frac{\partial v}{\partial z}, \quad w=0, \quad T=T_{w}, \quad C=C_{w} \quad \text { at } z=0, \\
u \longrightarrow 0, \quad v \longrightarrow 0, \quad T \longrightarrow T_{\infty}, \quad C \longrightarrow C_{\infty}, \quad P \longrightarrow P_{\infty} \quad \text { as } z \longrightarrow \infty
\end{gathered}
$$

Introducing a dimensionless normal distance from the disk, $\eta=z v^{-1 / 2}$ along with the following von Karman transformations, transforms the governing equations into a system of nonlinear ordinary differential equations:

$$
u=r f(\eta), \quad v=\operatorname{Rg}(\eta), \quad w=v^{1 / 2} h(\eta), \quad T=\theta(\eta), \quad C=\vartheta
$$

where $f, g, h, \theta$, and $\vartheta$ are nondimensional functions of modified dimensionless vertical coordinate $\eta$. The dimensionless temperature and concentration are $\theta=\left(T-T_{\infty}\right) /\left(T_{w}-T_{\infty}\right)$ and $\vartheta=\left(C-C_{\infty}\right) /\left(C_{w}-C_{\infty}\right)$.

We now have the following nonlinear ordinary differential equations:

$$
\begin{gathered}
f^{\prime \prime}-(f)^{2}+g^{2}-f^{\prime} h-M f=0, \\
g^{\prime \prime}-2 f g-h g^{\prime}-M g=0, \\
h^{\prime}+2 f=0, \\
\frac{1}{\operatorname{Pr}} \theta^{\prime \prime}-h \theta^{\prime}+\operatorname{MEc}\left(f^{2}+g^{2}\right)+\operatorname{Ec}\left(f^{\prime 2}+g^{\prime 2}\right)+\operatorname{Du} \vartheta^{\prime \prime}=0, \\
\frac{1}{\operatorname{Sc}} \vartheta^{\prime \prime}-h \vartheta^{\prime}+\operatorname{Sr} \theta^{\prime \prime}=0,
\end{gathered}
$$

where $M=\sigma B_{0}^{2} / \rho \Omega$ is the magnetic parameter, $\operatorname{Pr}=\rho v c_{p} / \kappa$ is the Prandtl number, Ec = $L^{2} \Omega^{2} R^{2} / c_{p}\left(T_{w}-T_{\infty}\right)$ is the Eckert number, Du $=D\left(C_{w}-C_{\infty}\right) k_{T} / c_{s} C_{p} v\left(T_{w}-T_{\infty}\right)$ is the Dufour number, $\mathrm{Sc}=v / D$ is the Schmidt number, $\mathrm{Sr}=D\left(T_{w}-T_{\infty}\right) k_{T} / v\left(C_{w}-C_{\infty}\right)$ is the Soret number. The continuity equation (2.1) is transformed into (2.11). Also the pressure term can be obtained from (2.4) as $P / \rho=v(\partial w / \partial z)-\left(w^{2} / 2\right)+$ constant, and this expression is then used to eliminate all the pressure terms [22]. The prime denotes derivative with respect to $\eta$. The transformed boundary conditions now become

$$
\begin{gathered}
f(0)=\gamma f^{\prime}(0), \quad g(0)=1+\gamma g^{\prime}(0), \quad h(0)=0, \quad \theta(0)=1, \quad \vartheta(0)=1, \\
f(\infty)=0, \quad g(\infty)=0, \quad \theta(\infty)=0, \quad \vartheta(0)=0,
\end{gathered}
$$

with $\gamma=[(2-\xi) \lambda] / \xi \mathcal{v}^{1 / 2}$ being the slip factor. The boundary conditions show that the radial component $f$, the tangential component $g$, temperature and concentration vanish sufficiently away from the rotating disk, whereas the axial velocity component $h$ is anticipated to approach an unknown asymptotic limit for large $\eta$-values. 


\section{Numerical Method of Solution}

In this section we present the numerical solution of the governing nonlinear systems of (2.9)(2.13). The equation system will be solved using the successive linearization method (SLM). The SLM was recently introduced as an efficient and robust method for solving boundary value problems [23]. In its basic application the SLM seeks to linearize the governing nonlinear differential equations to form an iterative system of linear differential equations which, in most cases, cannot be solved analytically. The Chebyshev pseudospectral method (or any other collocation method or numerical scheme) is then used to transform the iterative sequence of linearized differential equations into a system of linear algebraic equations which are converted into a matrix system. In this paper we present a compact general version of the SLM which can be applied to any system of nonlinear boundary value problems. We observe that (2.9)-(2.11) can be solved independently of (2.12)-(2.13). To generate a more general solution method, we introduce the following notation:

$$
y_{1}=f, \quad y_{2}=g, \quad y_{3}=h .
$$

Using the definition (3.1), the equation system (2.9)-(2.11) can be separated into its constituent linear and nonlinear components as

$$
a_{r r} y_{r}^{\prime \prime}+\sum_{k=1}^{3} b_{r k} y_{k}^{\prime}+\sum_{k=1}^{3} c_{r k} y_{k}+F_{r}\left(y_{1}, y_{2}, y_{3}\right)=0
$$

subject to

$$
\begin{gathered}
y_{1}(0)=r y_{1}^{\prime}(0), \quad y_{2}(0)=1+r y_{2}^{\prime}(0), \quad y_{3}(0)=0, \\
y_{1}(\infty)=0, \quad y_{2}(\infty)=0,
\end{gathered}
$$

where the coefficients $a_{r r}, b_{r k}, c_{r k}$ (for $r, k=1,2,3$ ) represent the constant factors of the various derivatives and $F_{r}\left(y_{1}, y_{2}, y_{3}\right)$ denotes the nonlinear components of (2.9)-(2.11) for $r=1,2,3$.

The SLM approach assumes that the solution of the system (3.2) can be expressed as

$$
y_{r}(\eta)=y_{r, s}(\eta)+\sum_{m=0}^{s-1} y_{r, m}(\eta)
$$

Starting from a suitable initial approximation $y_{r, 0}(t)$, the solution for $y_{r, s}(t)$ can be obtained by successively linearising equation (3.2) and solving the resulting linear system. The general form of the linearized equations for the SLM algorithm corresponding to (3.2) is

$$
\begin{gathered}
a_{r r} y_{r}^{\prime \prime}+\sum_{k=1}^{3} b_{r k} y_{k}^{\prime}+\sum_{k=1}^{3} c_{r k} y_{k}+F_{r}\left(y_{1}, y_{2}, y_{3}\right)=0 \\
a_{r r} y_{r, s}^{\prime \prime}+\sum_{k=1}^{3}\left(b_{r, k}+\frac{\partial F_{r}^{*}}{\partial y_{k}^{\prime}}\right) y_{k, s}^{\prime}+\sum_{k=1}^{3}\left(c_{r, k}+\frac{\partial F_{r}^{*}}{\partial y_{k}}\right) y_{k, s}=G_{r, s-1},
\end{gathered}
$$


subject to

$$
\begin{gathered}
r y_{1, s}^{\prime}(0)-y_{1, s}(0)=-\left(r \sum_{m=0}^{s-1} y_{1, m}^{\prime}(0)-\sum_{m=0}^{s-1} y_{1, m}(0)\right), \\
r y_{2, s}^{\prime}(0)-y_{2, s}(0)=-\left(r \sum_{m=0}^{s-1} y_{2, m}^{\prime}(0)-\sum_{m=0}^{s-1} y_{2, m}(0)+1\right), \\
y_{3, s}(0)=0, \quad y_{1, s}(\infty)=0, \quad y_{2, s}(\infty)=0,
\end{gathered}
$$

where

$$
\begin{gathered}
\frac{\partial F_{r}^{*}}{\partial y_{k}}=\frac{\partial F_{r}}{\partial y_{k}}\left(\bar{y}_{1}, \bar{y}_{2}, \bar{y}_{3}\right), \quad \text { with } \bar{y}_{r}=\sum_{m=0}^{s-1} y_{r, m}, \\
G_{r, s-1}=-\left[a_{r r} \bar{y}_{r}^{\prime \prime}+\sum_{k=1}^{3} b_{r k} \bar{y}_{k}^{\prime}+\sum_{k=1}^{3} c_{r k} \bar{y}_{k}+F_{r}\left(\bar{y}_{1}, \bar{y}_{2}, \bar{y}_{3}\right)\right] .
\end{gathered}
$$

The SLM algorithm (3.7) requires an initial approximation $y_{r, 0}$. This initial guess is chosen as a function that satisfies the boundary conditions (3.3). Suitable functions are

$$
y_{1,0}=0, \quad y_{2,0}=\frac{1}{1+\gamma} e^{-\eta}, \quad y_{3,0}=0
$$

Note that these initial guesses are chosen in such a way that they satisfy the boundary conditions. Thus, starting from the initial approximation (3.13), the solution of the linearized equation system (3.7) is solved iteratively using spectral collocation methods (or any other numerical method) for $y_{r, s}(\eta)$ (for $s=1,2,3, \ldots$ ). The approximate solution for each $y_{r}(\eta)$ is determined as the series solution

$$
y_{r}(\eta)=y_{r, 0}(\eta)+y_{r, 1}(\eta)+y_{r, 2}(\eta)+\cdots
$$

if

An SLM solution is said to be of order $i$ if the above series is truncated at $s=i$, that is,

$$
y_{r}(\eta)=\sum_{m=0}^{i} y_{r, m}(\eta)
$$

To solve the linearised system (3.7) we use the Chebyshev collocation spectral method in which the solution space is discretized using the Chebyshev-Gauss-Lobatto collocation point:

$$
\eta_{j}=\cos \left(\frac{\pi j}{N}\right), \quad j=0,1, \ldots, N,
$$


which are the extrema of the $N$ th order Chebyshev polynomial

$$
T_{N}(\eta)=\cos \left(N \cos ^{-1} \eta\right)
$$

Before applying the spectral method, it is convenient to transform the governing physical region $[0, \infty]$ for the problem to the interval $[-1,1]$ on which the spectral method is defined. This can be achieved by using the linear transformation $\tau=L(\eta+1) / 2$. Here, $L$ is chosen to be sufficiently large enough to numerically approximate infinity. The Chebyshev spectral collocation method (see, e.g., [24-26]) is based on the idea of introducing a differentiation matrix $D$ which is used to approximate the derivatives of the unknown variables $y_{r, s}$ at the collocation points as the matrix vector product:

$$
\frac{d y_{r, s}}{d \eta}=\sum_{k=0}^{N} \mathbf{D}_{j k} y_{r, s}\left(\tau_{k}\right)=\mathbf{D Y}_{r, s}, \quad j=0,1, \ldots, N
$$

where $\mathbf{D}=2 D / L$ and $\mathbf{Y}_{r, s}=\left[y_{r, s}\left(\tau_{0}\right), y_{r, s}\left(\tau_{1}\right), \ldots, y_{r, s}\left(\tau_{N}\right)\right]^{T}$ is the vector function at the collocation points $\tau_{j}$. The entries of $D$ can be computed in different ways. In this work we use the method proposed by Trefethen [25] in the cheb.m MATLAB m-file. If we denote the entries of the derivative matrix $\mathbf{D}$ by $\mathbf{D}_{j k}$, we can apply the spectral collocation method, with derivative matrices on the linear boundary value system (3.7) and boundary conditions (3.8)(3.10); we obtain the following linear matrix system:

$$
\mathbf{A}_{s-1} \mathbf{Y}_{s}=\mathbf{G}_{s-1}
$$

with the boundary conditions

$$
\begin{gathered}
r \sum_{k=0}^{N} D_{N k} y_{1, s}\left(\tau_{k}\right)-y_{1, s}\left(\tau_{N}\right)=\mathbf{p}_{s-1}, \\
r \sum_{k=0}^{N} D_{N k} y_{2, s}\left(\tau_{k}\right)-y_{2, s}\left(\tau_{N}\right)=\mathbf{q}_{s-1}, \\
y_{3, s}\left(\tau_{N}\right)=0, \quad y_{1, s}\left(\tau_{0}\right)=0, \quad y_{2, s}\left(\tau_{0}\right)=0,
\end{gathered}
$$

where $\mathbf{p}_{s-1}$ and $\mathbf{q}_{s-1}$ denote the right hand side of (3.8) and (3.9), respectively, when evaluated at the collocation points $\tau_{j}(j=0,2, \ldots, N)$. The vectors $\mathbf{Y}_{s}$ and $\mathbf{G}_{s-1}$ have dimension $3(N+$ 1) $\times 1$ and are defined as

$$
\mathbf{Y}_{s}=\left[Y_{1, s} ; Y_{2, s} ; Y_{3, s}\right]^{T}, \quad \mathbf{G}_{s-1}=\left[\mathbf{G}_{1, s-1} ; \mathbf{G}_{2, s-1} ; \mathbf{G}_{3, s-1}\right]^{T},
$$


with $Y_{r, s}$ and $\mathbf{G}_{r, s-1}$ being the vectors $y_{r, s 1}$ and $G_{r, s-1}(r=1,2,3)$, respectively, evaluated at the collocation points. The matrix $\mathbf{A}_{s-1}$ is an $3(N+1) \times 3(N+1)$ matrix that is defined as

$$
\mathbf{A}_{s-1}=\left[\begin{array}{lll}
A_{11} & A_{12} & A_{13} \\
A_{21} & A_{22} & A_{23} \\
A_{31} & A_{32} & A_{33}
\end{array}\right]
$$

with

$$
\mathbf{A}_{p q}= \begin{cases}a_{p p} \mathbf{D}^{2}+\left(b_{p p} \mathbf{I}+\frac{\partial F_{p}^{*}}{\partial y_{p}^{\prime}}\right) \mathbf{D}+c_{p p} \mathbf{I}+\frac{\partial F_{p}^{*}}{\partial y_{p}}, & p=q, \\ \left(b_{p q} \mathbf{I}+\frac{\partial F_{p}^{*}}{\partial y_{p}^{\prime}}\right) \mathbf{D}+c_{p q} \mathbf{I}+\frac{\partial F_{p}^{*}}{\partial y_{q}} \mathbf{I}, & p \neq q,\end{cases}
$$

where I is an identity matrix of order $N+1$. After imposing the boundary conditions (3.20)(3.22) on the matrix system (3.19), the solutions for $y_{r, s}(\eta)(s=1,2,3 \ldots)$ can be obtained by solving the iterative matrix system:

$$
\mathbf{Y}_{s}=\mathbf{A}_{s-1}^{-1} \mathbf{G}_{s-1} .
$$

The solutions for $f(\eta), g(\eta)$, and $h(\eta)$ obtained from (3.26) are substituted in (2.12) and (2.13) which now become a linear coupled system for the unknown variables $\theta$ and $\vartheta$. Applying the Chebyshev spectral collocation method on the resulting linear system, we obtain the following matrix system:

$$
\left[\begin{array}{cc}
\frac{1}{\operatorname{Pr}} \mathbf{D}^{2}-\mathbf{h D} & \mathrm{DuD} \\
\mathrm{SrD}^{2} & \frac{1}{\mathrm{Sc}} \mathbf{D}^{2}-\mathbf{h D}
\end{array}\right]\left[\begin{array}{l}
\tilde{\theta} \\
\tilde{\vartheta}
\end{array}\right]=\left[\begin{array}{l}
\mathbf{K} \\
\mathbf{O}
\end{array}\right]
$$

subject to the boundary conditions

$$
\begin{gathered}
\theta\left(\tau_{N}\right)=\vartheta\left(\tau_{N}\right)=1, \\
\theta\left(\tau_{0}\right)=\vartheta\left(\tau_{0}\right)=0,
\end{gathered}
$$

where

$$
\mathbf{K}=-\operatorname{MEc}\left(f^{2}+g^{2}\right)-\operatorname{Ec}\left(f^{\prime 2}+g^{\prime 2}\right)
$$

and $\mathbf{O}$ is a $(N+1) \times 1$ vector of zeros, $f, g, h$ are diagonal matrices corresponding to the solutions $f, g$, and $h$, respectively, when evaluated at the collocation points $\tau_{j}(j=$ $0,1,2, \ldots, N)$, and $\tilde{\theta}, \tilde{\vartheta}$ are the approximations of $\theta(\eta)$ and $\vartheta(\eta)$ at the collocation points. 


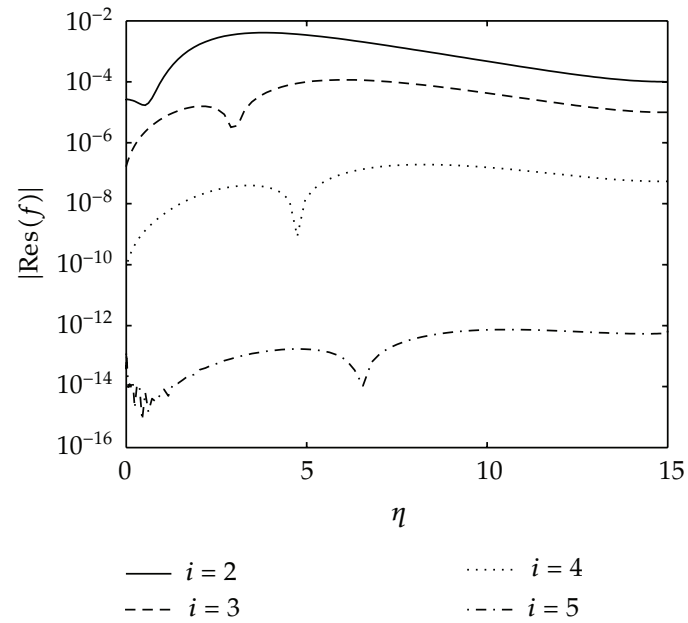

(a)

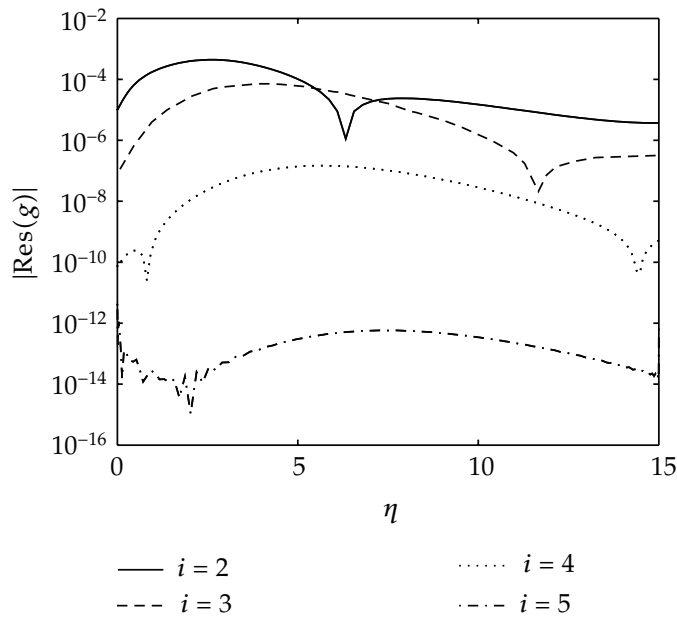

(b)

Figure 1: Res $f$ and Res $g$ for different order of approximation, when $\gamma=0.2, M=0.2$, $\operatorname{Pr}=0.71$, Sc $=$ $0.2, \mathrm{Sr}=1, \mathrm{Du}=0.06, \mathrm{Ec}=0.2$.

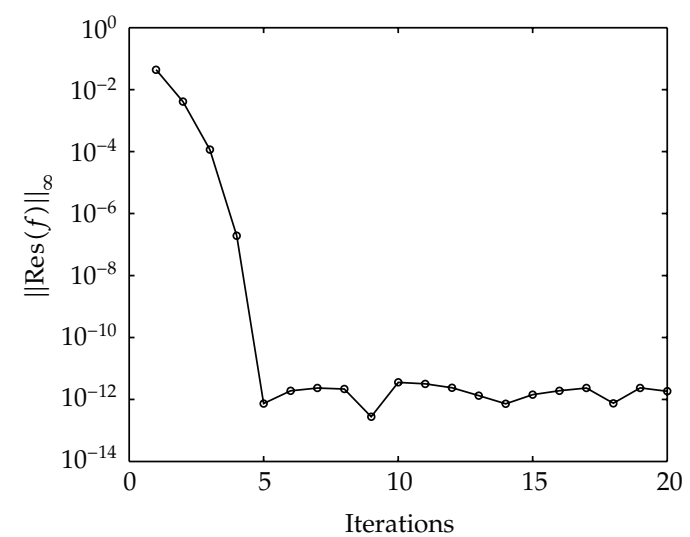

(a)

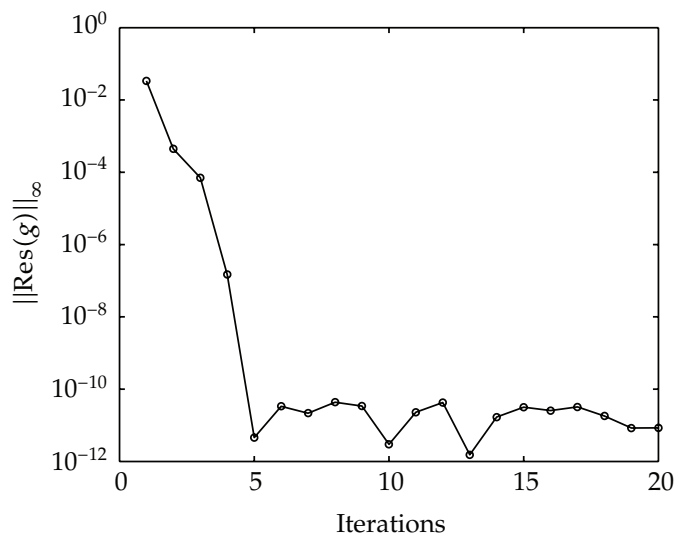

(b)

Figure 2: Norm of the residuals against iterations, when $\gamma=0.2, M=0.2, \operatorname{Pr}=0.71, \mathrm{Sc}=0.2, \mathrm{Sr}=1, \mathrm{Du}=$ $0.06, \mathrm{Ec}=0.2$.

After the boundary conditions (3.28) have been imposed on the matrix system (3.27), the resulting system can easily be inverted and solved as

$$
\left[\begin{array}{c}
\tilde{\theta} \\
\tilde{\vartheta}
\end{array}\right]=\left[\begin{array}{cc}
\frac{1}{\operatorname{Pr}} \mathbf{D}^{2}-\mathbf{h D} & \mathrm{DuD} \\
\mathrm{SrD}^{2} & \frac{1}{\mathrm{Sc}} \mathbf{D}^{2}-\mathbf{h D}
\end{array}\right]^{-1}\left[\begin{array}{l}
K_{1} \\
K_{2}
\end{array}\right] .
$$




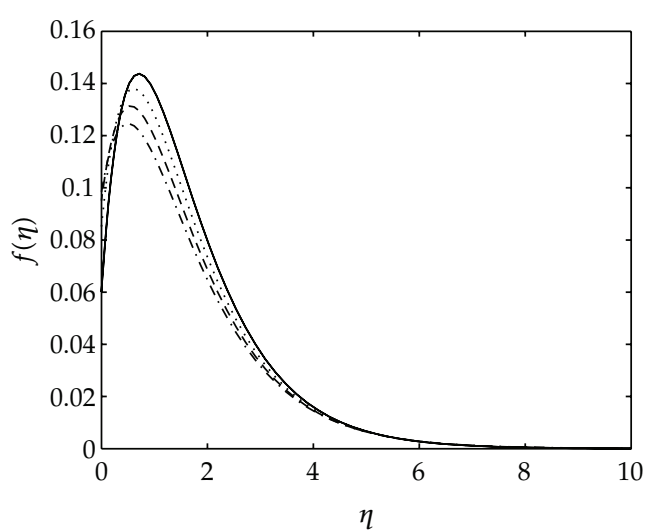

(a)

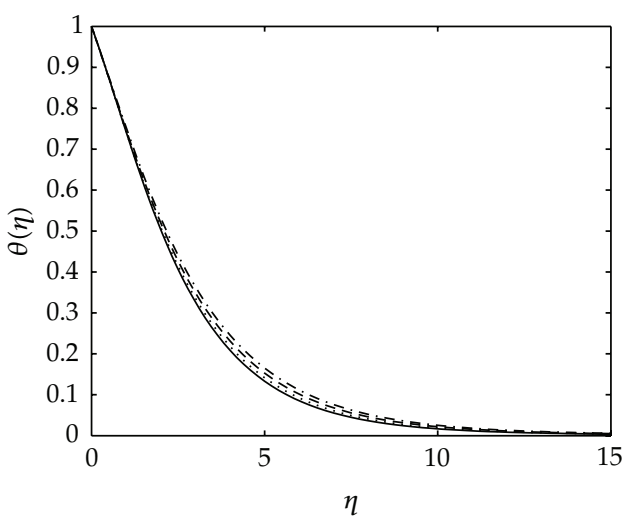

(c)

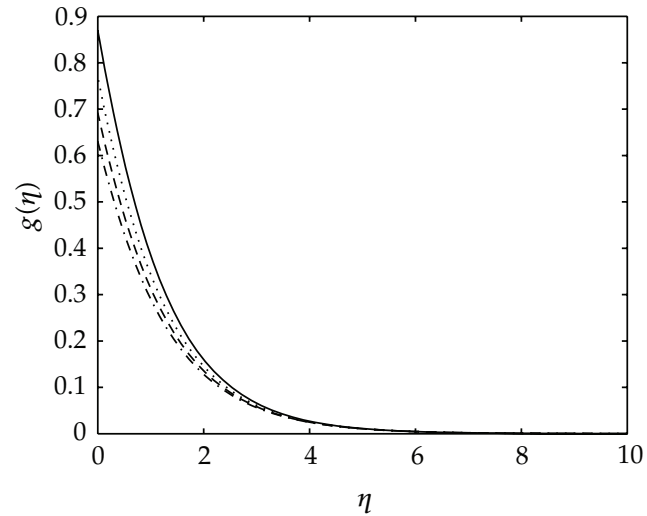

(b)

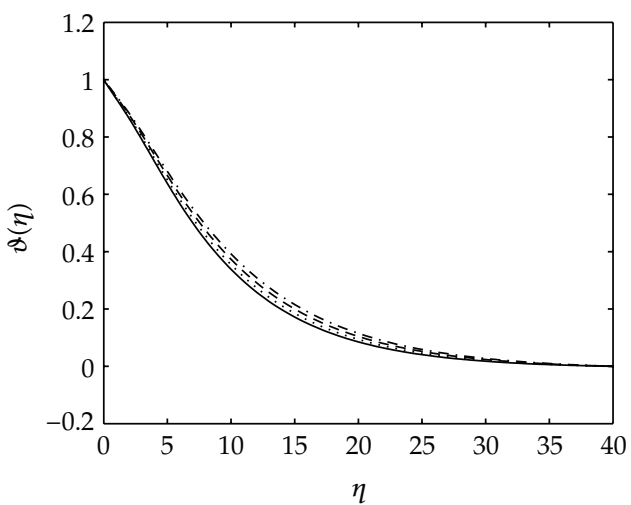

(d)

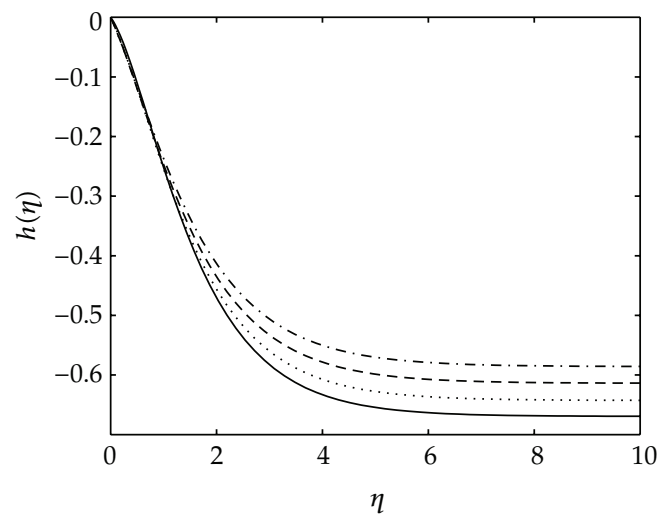

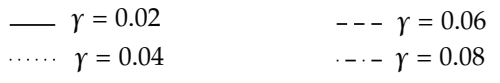

(e)

Figure 3: Sixth order SLM results for the variation of $\gamma$ on the flow properties when $M=0.2, \operatorname{Pr}=0.71, \mathrm{Sc}=$ $0.2, \mathrm{Sr}=1, \mathrm{Du}=0.06, \mathrm{Ec}=0.2$. 


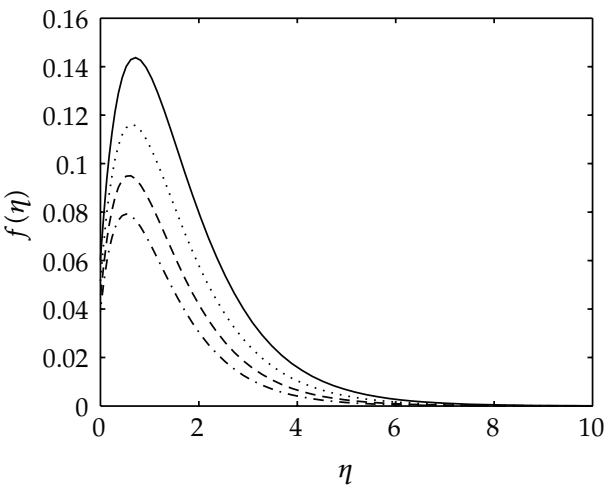

(a)

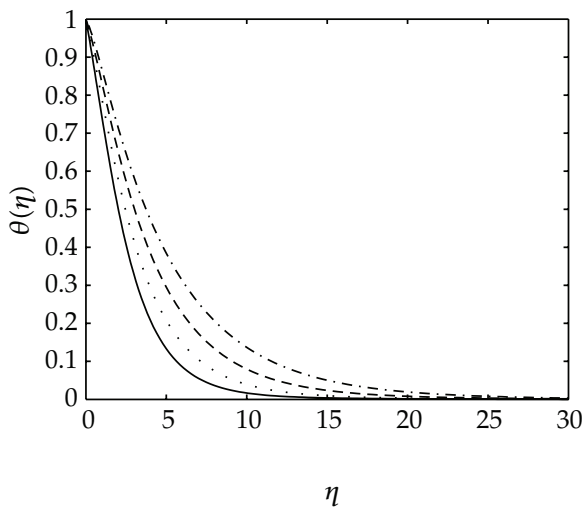

(c)

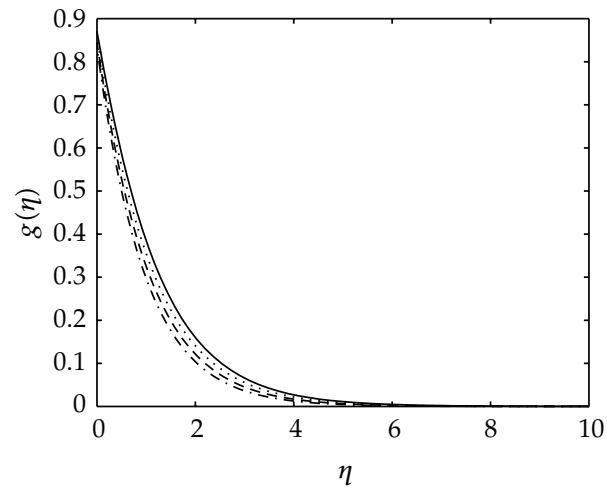

(b)

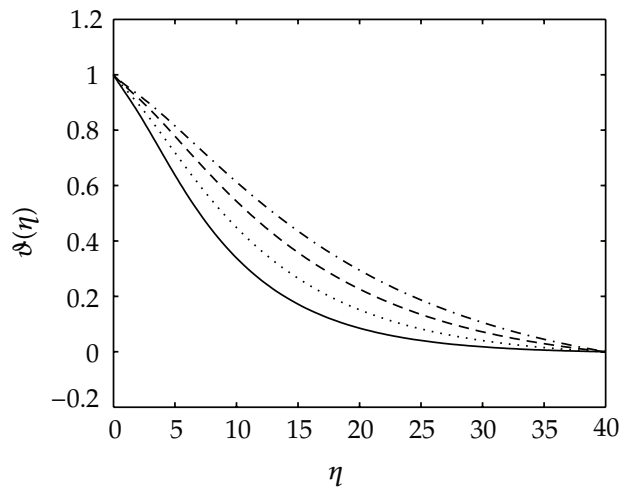

(d)

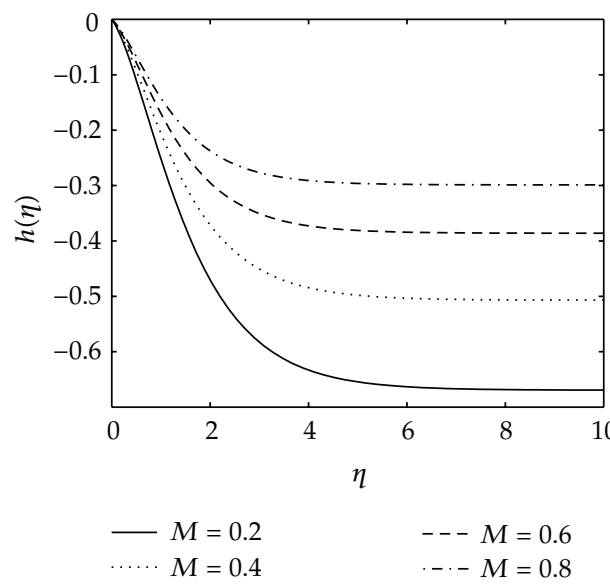

(e)

Figure 4: Sixth order SLM results for the variation of $M$ on the flow properties when $\gamma=0.2, \operatorname{Pr}=0.71, \mathrm{Sc}_{\mathrm{c}}=$ $0.2, \mathrm{Sr}=1, \mathrm{Du}=0.06, \mathrm{Ec}=0.2$. 


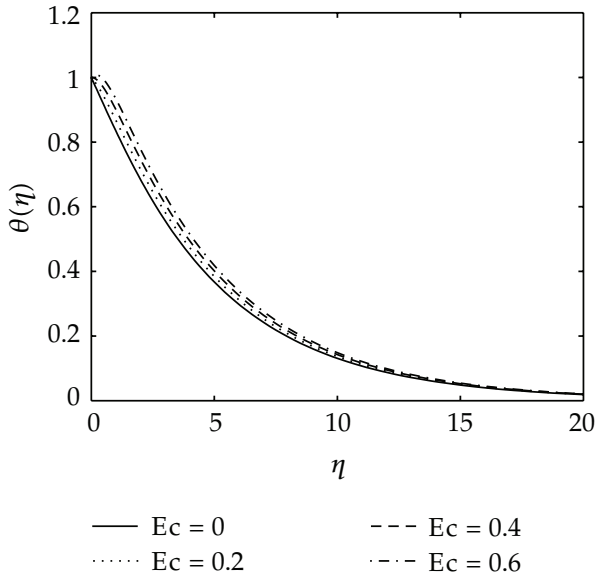

(a)

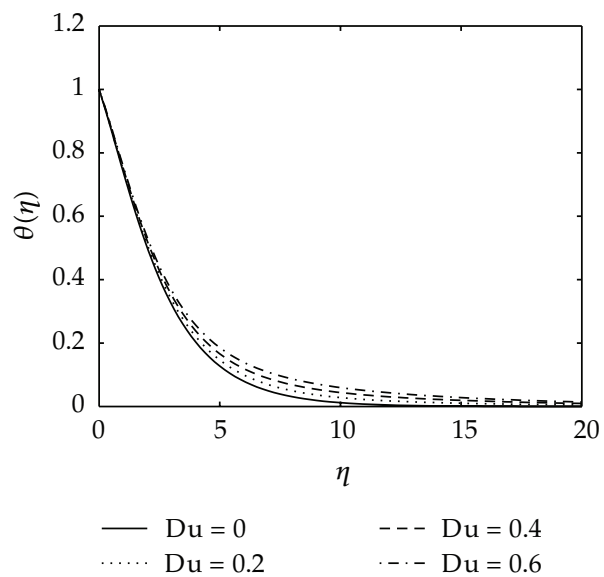

(b)

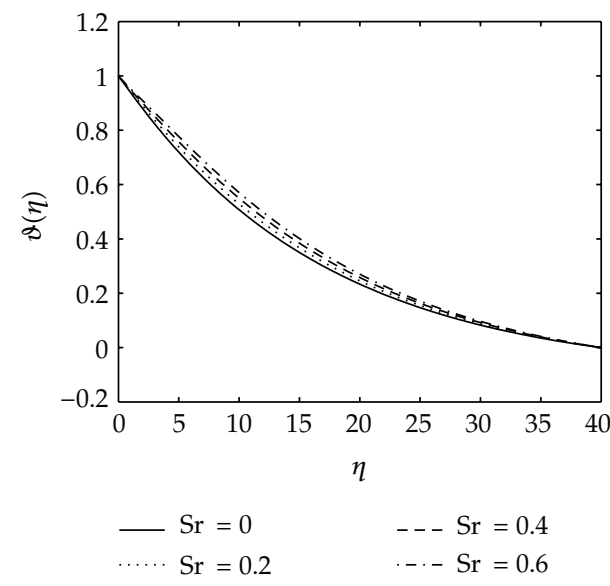

(c)

Figure 5: Effects of Ec and Du on temperature and also effects of Sc on concentration.

\section{Numerical Results}

The semianalytical results are obtained by solving (2.10)-(2.15) using the method elucidated in the previous section for various values of physical parameters to describe the physics of the problem. We remark that, unless otherwise specified, the SLM results presented in this analysis were obtained using $N=200$ collocation points, and $L=40$ was used as a numerical approximation infinity. To test the accuracy of the results we consider the residuals which are obtained by substituting the SLM approximate solutions and checking in the governing equations. The residuals corresponding to $f$ and $g$, for instance, are given by

$$
\begin{aligned}
& \operatorname{Res}(f) ; f^{\prime \prime}-(f)^{2}+g^{2}-f^{\prime} h-M f=0, \\
& \operatorname{Res}(g) ; g^{\prime \prime}-2 f g-h g^{\prime}-M g=0 .
\end{aligned}
$$


In Figure 1 we give the absolute value for the residual functions of $f$ and $g$, respectively, for different iterations. We observe that the residual becomes increasingly smaller as the number of iterations increases. We remark that the SLM algorithm was implemented using MATLAB which has a machine epsilon of 2.2204e - 16 which means that numbers are stored with about 15-16 digits of precision. We observe that after only 5 iterations, the residual error has almost converged to the machine epsilon of the computational software. This clearly shows the accuracy of the proposed method. We remark then that residual graphs of the same problem obtained using the homotopy analysis method (HAM) in [10] produced residuals values whose magnitude is significantly larger than the magnitude of the residuals depicted in Figure 1. This is the case even when the (HAM) iterations are increased to order 30. This indicates that the proposed SLM method is much more accurate than the HAM for the type of problems discussed in this paper. In Figure 2 we show the norm of the residual against the iterations. Again, it can be seen from this graph that as the iterations increase, the norm of the residual becomes smaller.

In Figures 3-5 we show the graphical representations of the numerical results when varying some of the governing parameters.

The effect of the slip factor $\gamma$ on the radial velocity $f(\eta)$, tangential velocity, $g(\eta)$, axial velocity $h(\eta)$, temperature $\theta(\eta)$, and concentration $\vartheta(\eta)$ profiles is shown in Figure 3 . In this investigation, we took Prandtt number of air $(\mathrm{Pr}=0.71)$ and Schmidt to be 0.2 . We observe in this figure that the radial velocity is reduced as the notating surface becomes more slippery. Also the shear-driven flow (tangential velocity) is reduced by the increasing values of the slip factor. However, towards the rotation disk, the axial velocity is increased as the slip factor increases. The centrifugal force associated with this circular motion causes the reductions of both the radial flow $f(\eta)$ and the shear-driven flow $g(\eta)$. In Figure 3, we also observe that the radial outflow $f(\eta)$ is compensated by an axial inflow $h(\eta)$ towards the rotating disk as $\gamma$ increases, in accordance with (2.11). We also observe that the magnitude of the temperature and concentration profiles slightly increase with an increase in a slip parameter.

Figure 4 depicts the effect of the magnetic field parameter $M$ on the radial velocity $f(\eta)$ profiles, tangential velocity profiles $g(\eta)$ profiles, axial velocity $h(\eta)$ profiles, temperature $\theta(\eta)$ profiles, and concentration $\vartheta(\eta)$.

It is observed in this figure that both the radial and tangential velocity profiles decrease with the increase of the magnetic field parameter values. This is because the presence of a magnetic field in an electrically conducting fluid introduces a force called Lorentz force which creates a drag-like force to slow down the flow along the disk surface. However, the Lorentz force accelerates flow in the axial direction causing the axial velocity profiles to increase as $M$ increases, (Alam and Ahammad [13], Motsa and Sibanda [27], and Shateyi et al. [28]). The temperature and concentration profiles increase as $M$ increases. This is because the drag-like forces caused by the pressure of a magnetic field cause both the thermal and solutal boundary layer to increase, hence increasing the temperature and concentration of the slowed down flow.

Figure 5 shows the effect of the Eckert number Ec on the temperature profiles and the effect on the Soret number $\mathrm{Sr}$ on the concentration profiles. The effect of increasing the values of the Eckert number is to enhance the temperature of the fluid at any point. This is expected according to (2.12). This is also because the heat energy is stored in the liquid due to frictional heating. The increased values of Ec lead to a strong viscous dissipation which significantly increases the temperature profiles, (Pal and Mondal [29]). The temperature of the fluid is also increased as the values of the Dufour parameter increase. Pal and Mondal [30] observed that the effect of increasing Du is to reduce the Nusselt number thereby causing the temperature 
profiles to increase as depicted in Figure 5 of the current investigation. We observe also in this figure that the concentration profiles increase with increasing Soret number values. This is because the increase of the values of $\mathrm{Sr}$ is caused by the increase of the temperature gradient which in turn leads to the increase of the concentration distributions with the fluid flow.

\section{Conclusion}

Numerical analysis has been carried out to investigate the MHD convective flow due to a rotating disk in the presence of viscous dissipation and ohmic heating, thermal-diffusion, and diffusion-thermo effects. The partial differential equations which describe the problem are transformed by using a suitable similarity transformation. The resultant nonlinear ordinary differential equations are then solved numerically using the SLM with the Chebyshev spectral collocation method. It was observed that the slip factor has significant effects on the fluid properties. The magnetic field helps to decelerate flow in the radial and tangential directions but accelerates flow in the axial direction. It also increases the temperature and concentration distributions. Both thermal diffusion and diffusion-thermo have some significant effect on the concentration and temperature profiles.

\section{Acknowledgments}

The authors wish to acknowledge financial support from the University of Venda and the National Research Foundation (NRF).

\section{References}

[1] T. von Karman, "Uber laminare und turbulente Reibung," Zeitschrift für Angewandte Mathematik und Mechanik, vol. 1, no. 4, pp. 233-255, 1921.

[2] W. G. Cochran, "The flow due to a rotating disk," Proceedings of the Cambridge Philosophical Society, vol. 30, no. 3, pp. 365-375, 1934.

[3] E. R. Benton, "On the flow due to a rotating disk," The Journal of Fluid Mechanics, vol. 24, pp. 781-800, 1966.

[4] H. A. Attia, "Unsteady flow and heat transfer of viscous incompressible fluid with temperaturedependent viscosity due to a rotating disc in a porous medium," Journal of Physics A, vol. 39, no. 4, pp. 979-991, 2006.

[5] M. Miklavčič and C. Y. Wang, "The flow due to a rough rotating disk," Zeitschrift für Angewandte Mathematik und Physik, vol. 55, no. 2, pp. 235-246, 2004.

[6] A. Arikoglu and I. Ozkol, "On the MHD and slip flow over a rotating disk with heat transfer," International Journal of Numerical Methods for Heat and Fluid Flow, vol. 16, no. 2, pp. 172-184, 2006.

[7] N. G. Kafoussias and E. W. Williams, "Thermal-diffusion and diffusion-thermo effects on mixed freeforced convective and mass transfer boundary layer flow with temperature dependent viscosity," International Journal of Engineering Science, vol. 33, no. 9, pp. 1369-1384, 1995.

[8] D. A. Siginer, "Thermal science and engineering with emphasis on porous media(forward)," Journal of Applied Mechanics, vol. 73, pp. 1-4, 2006.

[9] E. Osalusi, J. Side, and R. Harris, "Thermal-diffusion and diffusion-thermo effects on combined heat and mass transfer of a steady MHD convective and slip flow due to a rotating disk with viscous dissipation and Ohmic heating," International Communications in Heat and Mass Transfer, vol. 35, no. 8, pp. 908-915, 2008.

[10] M. M. Rashidi, T. Hayat, E. Erfani, S. A. Mohimanian Pour, and A. A. Hendi, "Simultaneous effects of partial slip and thermal-diffusion and diffusion-thermo on steady MHD convective flow due to a rotating disk," Communications in Nonlinear Science and Numerical Simulation, vol. 16, no. 11, pp. 4303-4317, 2011. 
[11] M. Turkyilmazoglu, "Analytic approximate solutions of rotating disk boundary layer flow subject to a uniform suction or injection," International Journal of Mechanical Sciences, vol. 52, no. 12, pp. 1735-1744, 2010.

[12] S. Shateyi, S. S. Motsa, and P. Sibanda, "The effects of thermal radiation, hall currents, soret, and dufour on MHD flow by mixed convection over a vertical surface in porous media," Mathematical Problems in Engineering, vol. 2010, Article ID 627475, 20 pages, 2010.

[13] M. S. Alam and M. U. Ahammad, "Effects of variable chemical reaction and variable electric conductivity on free convective heat and mass transfer flow along an inclined stretching sheet with variable heat and mass fluxes under the influence of Dufour and Soret effects," Nonlinear Analysis: Modelling and Control, vol. 16, no. 1, pp. 1-16, 2011.

[14] D. Srinivasacharya and K. Kaladhar, "Analytical solution of MHD free convective flow of couple stress fluid in an annulus with Hall and Ion-slip effects," Nonlinear Analysis: Modelling and Control, vol. 16, no. 4, pp. 477-487, 2011.

[15] D. Pal and H. Mondal, "MHD non-Darcian mixed convection heat and mass transfer over a nonlinear stretching sheet with Soret-Dufour effects and chemical reaction," International Communications in Heat and Mass Transfer, vol. 38, no. 4, pp. 463-467, 2011.

[16] M. Dehghan, J. M. Heris, and A. Saadatmandi, "Application of semi-analytic methods for the Fitzhugh-Nagumo equation, which models the transmission of nerve impulses," Mathematical Methods in the Applied Sciences, vol. 33, no. 11, pp. 1384-1398, 2010.

[17] S. Liao, Beyond Perturbation: Introduction to Homotopy Analysis Method, vol. 2, Chapman \& Hall/CRC, Boca Raton, Fla, USA, 2003.

[18] M. Dehghan and R. Salehi, "Solution of a nonlinear time-delay model in biology via semi-analytical approaches," Computer Physics Communications, vol. 181, no. 7, pp. 1255-1265, 2010.

[19] S. S. Motsa, P. Sibanda, and S. Shateyi, "A new spectral-homotopy analysis method for solving a nonlinear second order BVP," Communications in Nonlinear Science and Numerical Simulation, vol. 15, no. 9, pp. 2293-2302, 2010.

[20] S. S. Motsa, S. Shateyi, G.T. Marewo, and P. Sibanda, "An improved spectral homotopy analysis method for MHD flow in a semi-porous channel," Numerical Algorithms, vol. 60, no. 3, pp. 463-481, 2012.

[21] S. S. Motsa and S. Shateyi, "Successive linearisation solution of free convection non-Darcy flow with heat and mass transfer," in Advanced Topics in Mass Transfer, M. El-Amin, Ed., pp. 425-438, InTech, 2011.

[22] F. M. Ali, R. Nazar, and N. M. Arifin, "MHD viscous flow and heat transfer induced by a permeable shrinking sheet with prescribed surface heat flux," WSEAS Transactions on Mathematics, vol. 9, no. 5, pp. 365-375, 2010.

[23] S. S. Motsa, "New algorithm for solving non-linear BVPs in heat transfer," International Journal of Modeling, Simulation E Scientific Computing, vol. 2, no. 3, pp. 355-373, 2011.

[24] C. Canuto, M. Y. Hussaini, A. Quarteroni, and T. A. Zang, Spectral Methods in Fluid Dynamics, Springer, Berlin, Germany, 1988.

[25] L. N. Trefethen, Spectral Methods in MATLAB, Society for Industrial and Applied Mathematics, 2000.

[26] J. A. C. Weideman and S. C. Reddy, "A MATLAB differentiation matrix suite," ACM Transactions on Mathematical Software, vol. 26, no. 4, pp. 465-519, 2000.

[27] S. S. Motsa and P. Sibanda, "On the solution of MHD flow over a nonlinear stretching sheet by an efficient semi-analytical technique," International Journal for Numerical Methods in Fluids, vol. 68, no. 12, pp. 1524-1537, 2012.

[28] S. Shateyi, P. Sibanda, and S. S. Motsa, "Magnetohydrodynamic flow past a vertical plate with radiative heat transfer," Journal of Heat Transfer, vol. 129, no. 12, pp. 1708-1713, 2007.

[29] D. Pal and H. Mondal, "Hydromagnetic non-Darcy flow and heat transfer over a stretching sheet in the presence of thermal radiation and Ohmic dissipation," Communications in Nonlinear Science and Numerical Simulation, vol. 15, no. 5, pp. 1197-1209, 2010.

[30] D. Pal and H. Mondal, "MHD non-Darcy mixed convective diffusion of species over a stretching sheet embedded in a porous medium with non-uniform heat source/sink, variable viscosity and Soret effect," Communications in Nonlinear Science and Numerical Simulation, vol. 17, no. 2, pp. 672-684, 2012. 


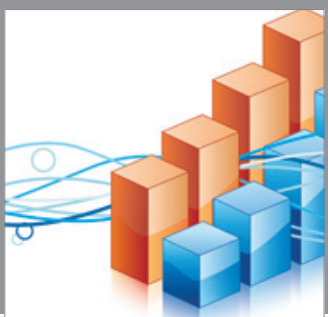

Advances in

Operations Research

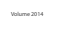

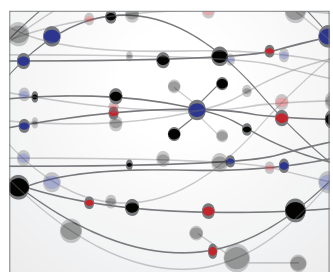

\section{The Scientific} World Journal
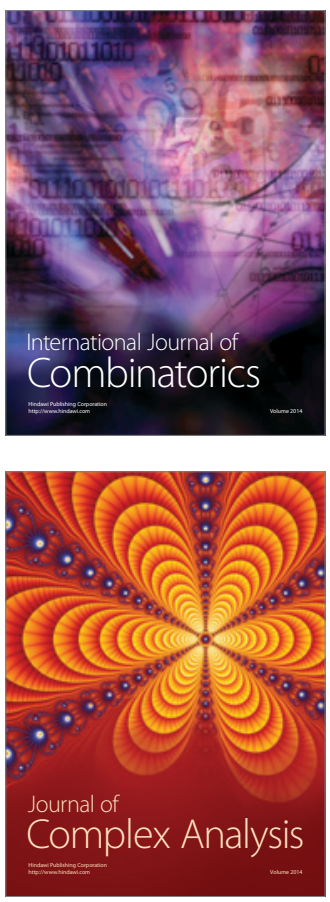

International Journal of

Mathematics and

Mathematical

Sciences
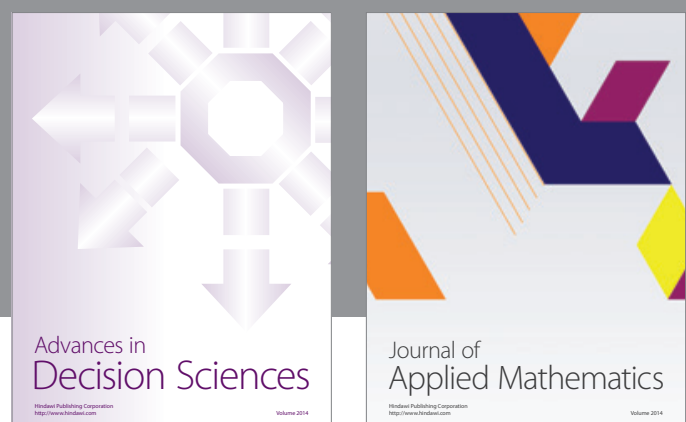

Journal of

Applied Mathematics
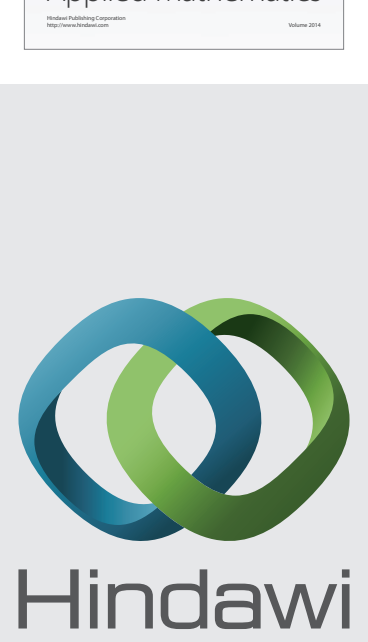

Submit your manuscripts at http://www.hindawi.com
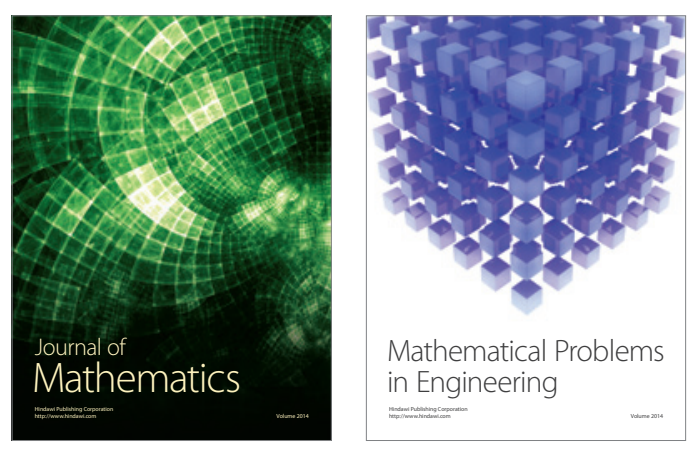

Mathematical Problems in Engineering
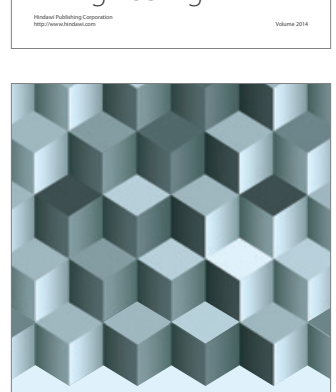

Journal of

Function Spaces
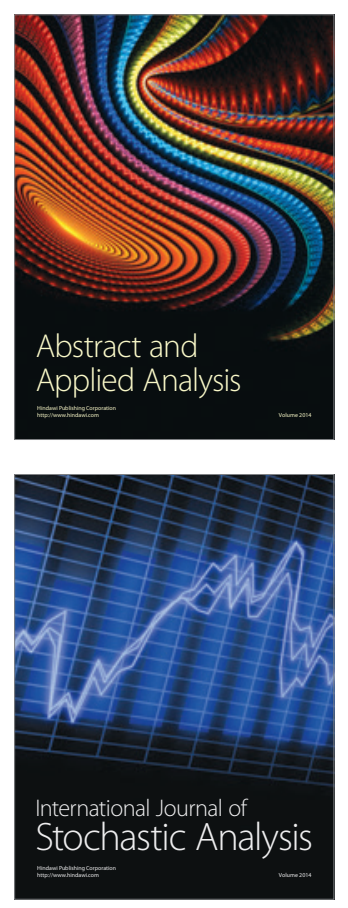

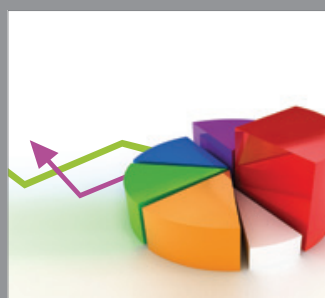

ournal of

Probability and Statistics

Promensencen
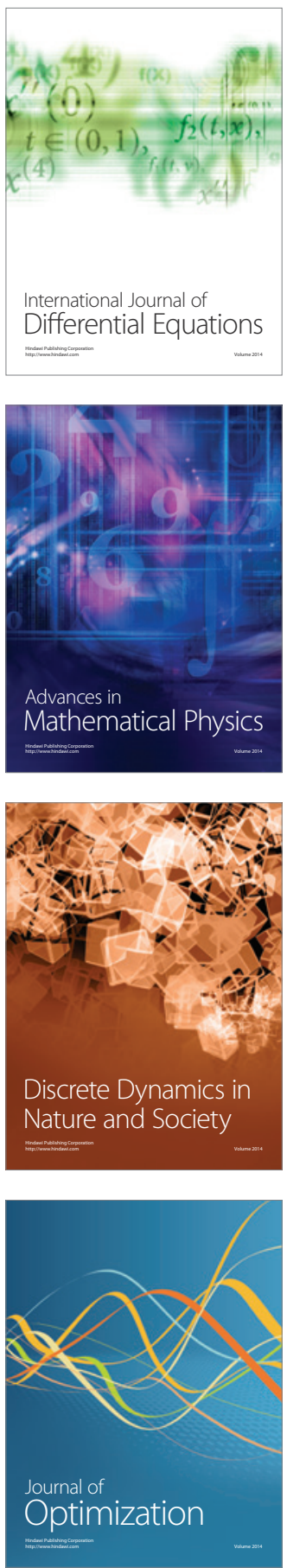